\title{
Selecting Effective Herbal Medicines for Attention-Deficit/Hyperactivity Disorder via Text Mining of Donguibogam
}

\author{
Hyo Won Bae,' Se Yeon Lee, ${ }^{1}$ Sung Ji Kim, ${ }^{1}$ Hwa Kyoung Shin $\mathbb{D}^{2}$, \\ Byung Tae Choi $\mathbb{D}^{2},{ }^{2}$ and Jin Ung Baek $\mathbb{D}^{1}$ \\ ${ }^{1}$ Division of Humanities and Social Medicine, School of Korean Medicine, Pusan National University, \\ Yangsan 626-870, Republic of Korea \\ ${ }^{2}$ Division of Meridian and Structural Medicine, School of Korean Medicine, Pusan National University, \\ Yangsan 626-870, Republic of Korea
}

Correspondence should be addressed to Jin Ung Baek; mukjagan@pusan.ac.kr

Received 18 April 2019; Accepted 20 May 2019; Published 4 June 2019

Academic Editor: Armando Zarrelli

Copyright (C) 2019 Hyo Won Bae et al. This is an open access article distributed under the Creative Commons Attribution License, which permits unrestricted use, distribution, and reproduction in any medium, provided the original work is properly cited.

\begin{abstract}
Objective. Several attempts have been made to reduce the harmful side effects and increase the efficacy of current drugs used to treat attention-deficit/hyperactivity disorder (ADHD). Many articles have studied medicinal herbs as an effective supplement in treating ADHD. In a similar manner, this study provides foundational data to identify herbs that are potentially effective in treating ADHD by text mining of Donguibogam, which is a comprehensive summation of the important traditional principles and practices of Korean medicine. Methods. Text mining was performed for 3833 herbal prescriptions and 1108 medicinal herbs comprising prescriptions listed in Donguibogam. The first step was frequency analysis followed by chi-square test, which is a statistical hypothesis test. Results and Conclusions. Twelve medicinal herbs were selected for each ADHD subtype: hyperactivity $\mathrm{ADHD}$ type (ADHD-PHI) and attention-deficit ADHD type (ADHD-PI). Compared to previous research on traditional literature, a newer and more efficient methodology of selecting herbal medicines was developed in this process.
\end{abstract}

\section{Introduction}

Patients with attention-deficit/hyperactivity disorder (ADHD) present with symptoms such as lack of concentration, impulsiveness, and excessive activity, and ADHD is one of the neurobehavioral disorders commonly occurring in childhood. [1].

Currently, approximately 5-7\% of school-aged children and $2-5 \%$ of adults are believed to have ADHD worldwide $[2,3]$. In the United States, the percentage of ADHD patients among children and adolescents was 6.1\% (Diagnostic and Statistical Manual of Mental Disorders [DSM]-IV) in 1998 and $10.2 \%$ (DSM-V) in 2016 [4]. Some argue that with the development of diagnostic methods, the number of ADHD patients in the United States increased over the years, and a similar increase occurred in many European countries [57]. In Korea, a less than $1 \%$ diagnostic rate was reported in a study using actual hospital treatment records, but $5.9 \%$ of children with ADHD were reported in another study using the random sampling method in elementary school students $[8,9]$. This indicated that although a sizable portion of the population experienced ADHD symptoms, they have not visited the hospital for diagnosis.

Both genetic and environmental factors affect the occurrence of ADHD, but the exact cause has not yet been elucidated. However, some researchers have found that symptoms of impulsiveness and attention-deficiency tend to be present when the function of the frontal lobe of controlling thought and concentration decreases. Another possible reason could be a problem in metabolizing neurotransmitters such as catecholamine, norepinephrine, and dopamine [10]. Therefore, stimulants of the central nervous system (methylphenidate and pemoline), norepinephrine reuptake inhibitors (atomoxetine), or antidepressants are mainly used as treatment 
drugs [11]. Approximately $90 \%$ of Korean ADHD patients were prescribed methylphenidate, because drugs approved for use in Korea are limited and pemoline has been linked to hepatotoxicity [12]. However, side effects of methylphenidate include decreased appetite, insomnia, growth delay, tic disorder, nausea, stomachache, headache, and fatigue $[13,14]$. In preschool children below 7 years of age in particular, $30 \%$ of them were more likely to suffer growth delay compared to school-age children, which meant that they experienced more severe side effects [15]. To avoid such harmful side effects, natural and holistic treatments using Korean medicine need to be considered as an alternative.

Thus far, traditional Korean medicine or traditional Chinese medicine research related to ADHD has been mainly composed of research based on original literature, systematic review based on search treatises, and experimental research testing the treatment effect of herbs on ADHD [16-21]. However, research based on original literature was limited to only listing herbal medicines that frequently appeared in text. On the other hand, experimental research had difficulty in selecting the right herbal medicines to test. To overcome these limitations, this study preselected a list of herbal medicines that statistically significantly deemed potentially effective for ADHD by screening Donguibogam, which is a comprehensive compilation of important traditional Eastern medicines. This study played the role of a preliminary experiment to not only save time and money, but also increase the success rate of future experimental research.

Furthermore, this study considered hyperactivity ADHD type (ADHD-PHI) and attention-deficit ADHD type (ADHD-PI) as two separate disease symptoms. DSM is the most typical diagnostic method of ADHD, which classifies ADHD into three subtypes: predominantly inattentive (ADHD-PI), predominantly hyperactive-impulsive (ADHD$\mathrm{PH}$ ), and combined type (ADHD-C) [22]. In Western medicine, symptoms of ADHD are heavily linked to the frontal lobe of the brain or neurotransmitters, and the same drugs (such as central nervous system stimulants, norepinephrine reuptake inhibitors, or antidepressants) are used to treat both ADHD-PI and ADHD-PHI. However, traditional Korean medicine uses symptomatic treatment that pays close attention to each symptom. Therefore, this study found medicinal herbs specific for each subtype (ADHD-PHI and ADHD-PI) and also listed herbs that could be applied to both.

\section{Materials and Methods}

The herbal prescriptions deemed potentially effective for ADHD in Donguibogam (ADHD PEHP) were compared to the remaining herbal prescriptions in the book using the chi-square test, a statistical method used to determine homogeneity between two groups for a specific variable. Thus, the authors were able to select only those herbs showing statistically significant efficacy.

2.1. Generating Baseline Data for Analysis. First, all prescriptions in Donguibogam were extracted, and the herbs comprising each prescription were listed. In this process, medicinal herbs with synonymous names were standardized under one name.

Second, Korean medical symptom names corresponding to ADHD-PHI and ADHD-PI were defined using existing research, and prescriptions and herbs containing at least one of these symptom names were selected (Figure 1) [23]. Information regarding prescriptions and herbs in Donguibogam was extracted from a state-run website called the Korean traditional knowledge portal (http://www.koreantk .com/ktkp2014).

\section{Symptom Names in Traditional Korean Medicine That Matched ADHD}

(i) ADHD-PHI: Jingji, zhengchong, zhanyu, xu fan, fan zao, yin xuhuo dong, and shanghan $\mathrm{xu}$ xuezheng (searched by Xu Xue)

(ii) ADHD-PI: Jianwang, laojuan shang, qi fa, and yin xu neire

2.2. Statistical Analysis. The frequency of occurrence was calculated for each medicinal herb in ADHD-PHI PEHP. Only those herbs that made up more than $2 \%$ of the total were selected as the experimental group (Table 1).

Among these, "Cinnabaris" was removed because it is a medicinal herb derived from minerals and its use is restricted. Additionally, the control group was selected by counting the number of times the herbs that were selected as the experimental group appeared in the rest of the Donguibogam prescriptions, except for ADHD-PHI PEHP. The same steps were repeated for the prescriptions potentially effective for ADHD-PI. Following this, a chi-squared test was performed to evaluate whether experimental group herbs were more effective in the treatment of ADHD-PHI or ADHD-PI in a statistically significant manner than control group herbs (Figures 2 and 3$)(\mathrm{P}<0.05)$. However, in the chi-squared test, Yate's continuity correction was used when the expected frequency was 5-9, and Fisher's exact test was used when it was under 5 for more than 20\% of the cells [24]. For data analysis, Microsoft Excel 2016 was used to generate baseline data, and SAS version 9.4 was used for statistical analysis.

\section{Results}

3.1. ADHD-PHI. For symptoms of ADHD-PHI, the top 5 herbs with the highest composition rate were Poria cocos (P.C, 6.69\%), Glycyrrhiza uralensis (G.V, 6.50\%), Rehmannia glutinosa (R.G, 4.59\%), Angelica gigas (A.G, 4.49\%), and Panax ginseng (P.G, 4.21\%) (Figure 4).

Compared to the rest of the prescriptions, other than those of ADHD-PHI PEHP, the usage of P.C, G.V, R.G, A.G, P.G, Liriope platyphylla (L.P), Paeonia lactiflora (P.L), Ziziphus jujuba (Z.J), Coptis chinensis (C.C), Polygala tenuifolia (P.T), and Phellodendron amurense (P.A) for ADHD-PHI was statistically significant at 0.01 and that of Pinellia ternata (P.M) was significant at 0.05 . The usage of Atractylodes lancea (A.L), Citrus reticulata (C.R), and Cnidium officinale (C.O) was not statistically significant for ADHD-PHI. In cases of L.P and C.O, Yate's continuity correction chi-squared value 
TABLE 1: Medicinal herbs selected as the experimental group ( $\mathrm{n}=18)$.

\begin{tabular}{lccc}
\hline Name & Author & Part of the plant & Abbreviation \\
\hline Acorus calamus & L. & rhizoma & root \\
\hline Angelica gigas & Nakai & rhizoma & A.C \\
\hline Atractylodes lancea & (Thunb.) DC. & root & A.L \\
\hline Astragalus membranaceus & (Fisch.) Bunge & root & A.M \\
\hline Bupleurum chinense & DC. & rhizoma & B.C \\
\hline Coptis chinensis & Franch. & rhizoma & C.C \\
\hline Cnidium officinale & Makino & peel & C.O \\
\hline Citrus reticulata & Blanco & root & tuber \\
\hline Glycyrrhiza uralensis & Fisch. & cortex & \\
\hline Liriope platyphylla & F.T.Wang et Tang & C.R \\
\hline Phellodendron amurense & Rupr. & G.V \\
\hline Poria cocos & Wolf & root \\
\hline Panax ginseng & C.A.Mey. & root \\
\hline Paeonia lactiflora & Pall. & tuber \\
\hline Pinellia ternata & (Thunb.) Makino & root & P.A \\
\hline Polygala tenuifolia & Willd. & root & P.C \\
\hline Rehmannia glutinosa & (Gaertn.) DC. & seed \\
\hline Ziziphus jujuba var. spinosa & (Bunge) Hu ex H.F.Chow & P.L \\
\hline
\end{tabular}

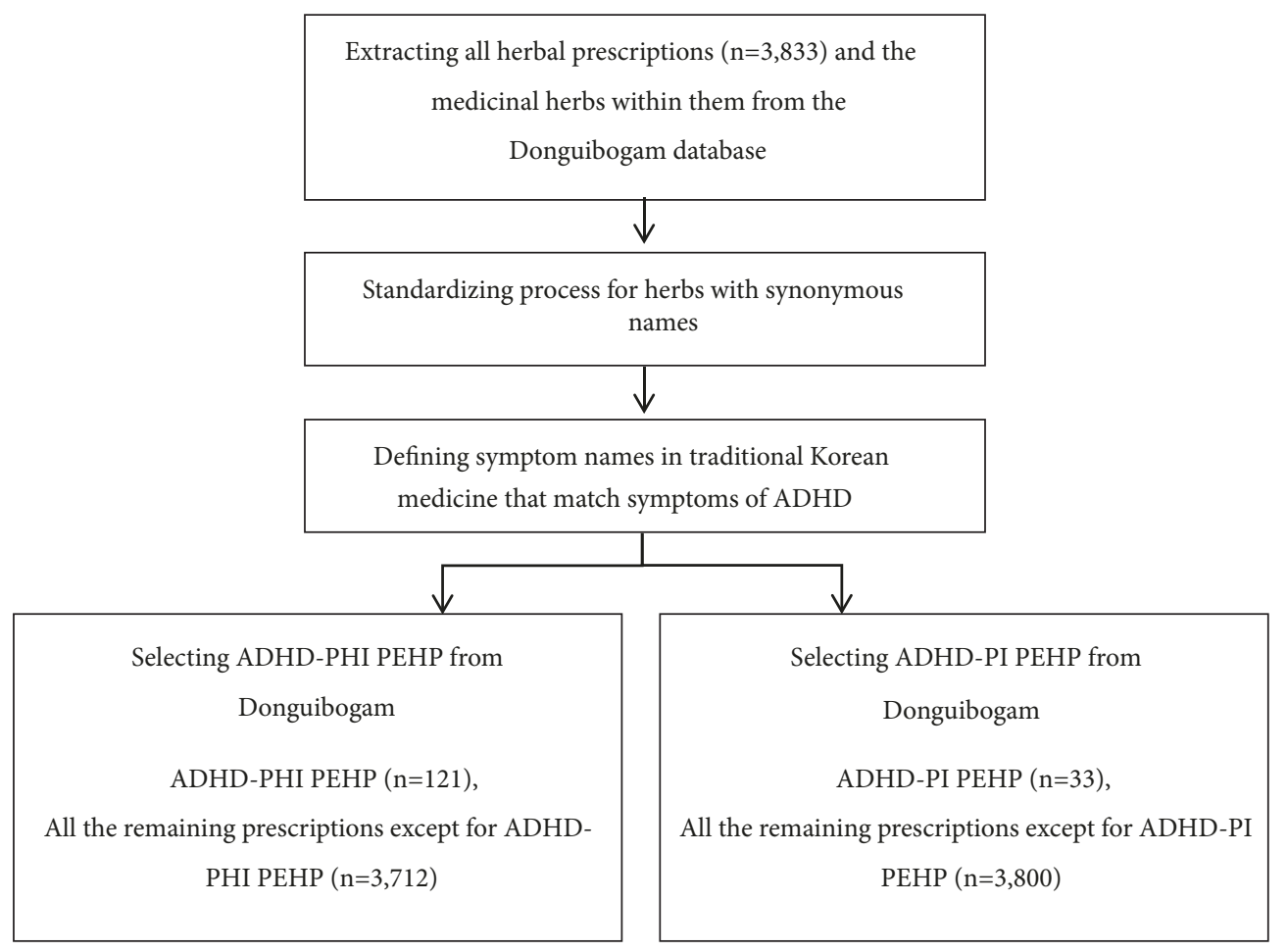

*ADHD-PHI (or PI) PEHP: Herbal prescriptions that are potentially effective for attention-deficit/hyperactivity disorder (ADHD-PHI or PI) type according to Donguibogam

FIGURE 1: Selecting herbal prescriptions deemed potentially effective for attention-deficit/hyperactivity disorder in Donguibogam (ADHD PEHP). 


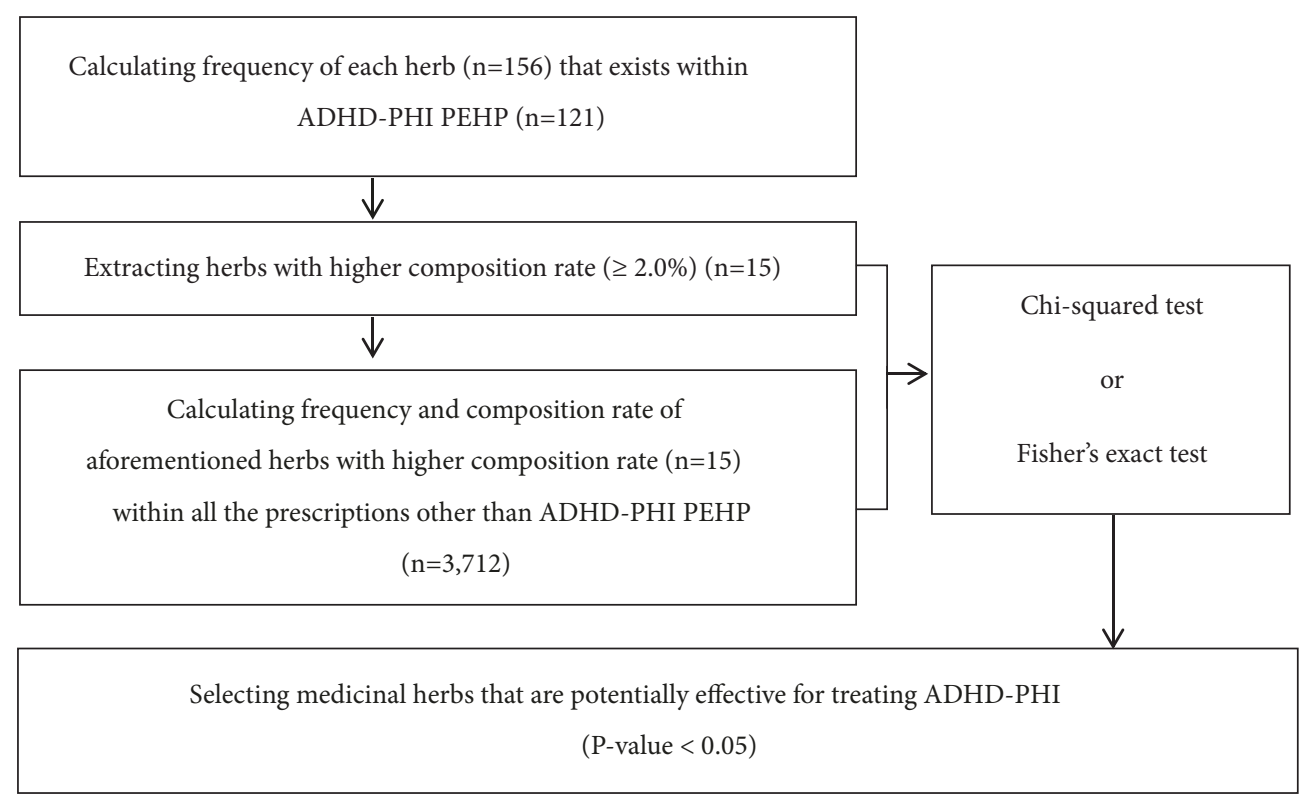

FIGURE 2: Statistical stage of selecting herbs deemed potentially effective for hyperactivity attention-deficit/hyperactivity disorder type.

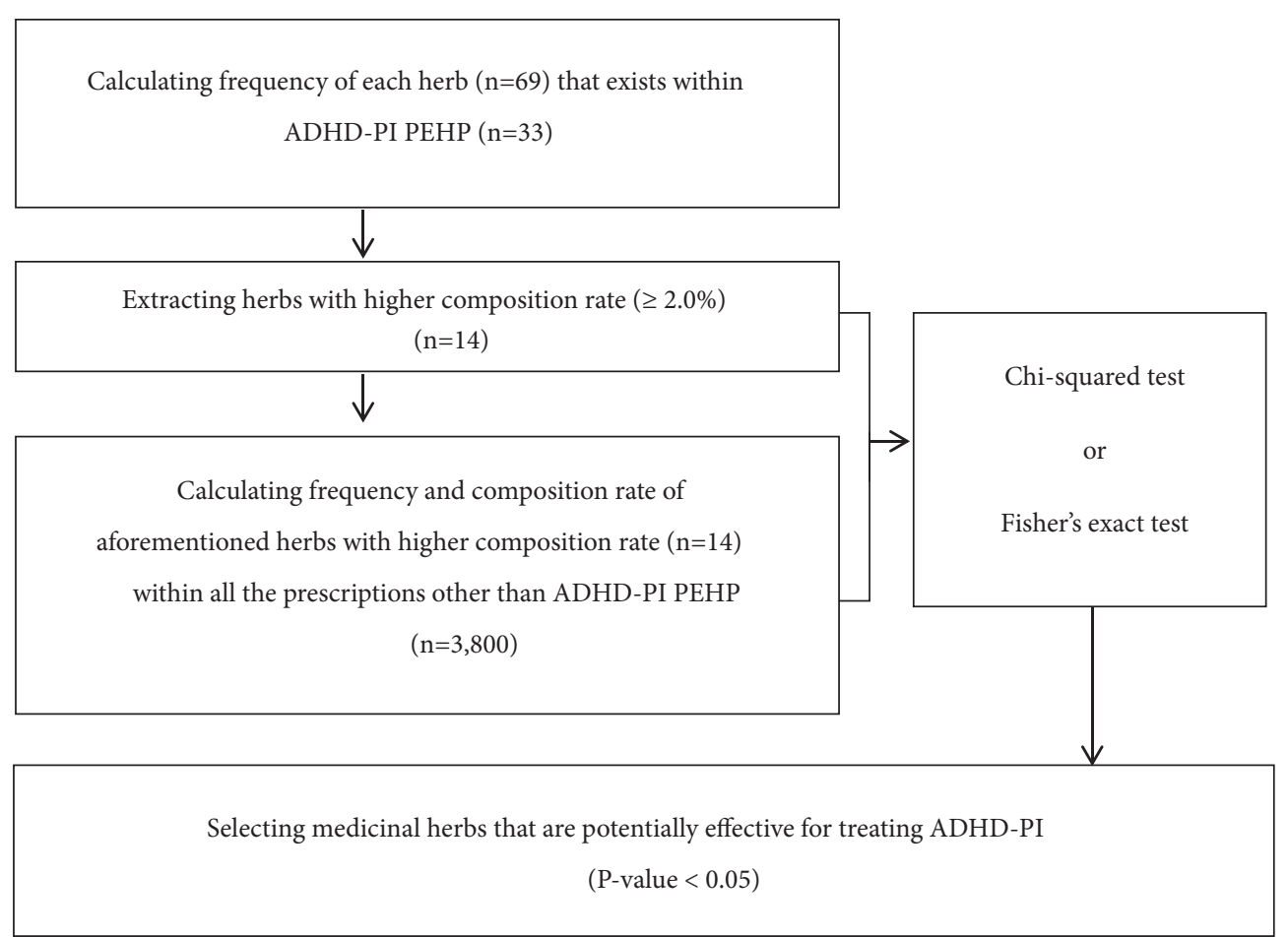

FIGURE 3: Statistical stage of selecting herbs deemed potentially effective for attention-deficit attention-deficit/hyperactivity disorder type.

was used, while the Fisher's exact test was used for Z.J and P. T. Based on their chi-squared values, L.P (127.12), P.C (83.29), and R.G (47.41) were found to have been used specifically for ADHD-PHI (in that order of specificity). The chi-squared values of Z.J (349.09) and P.T (102.80) were very high, but the authors decided to exclude them from the ranking order of significance because $25 \%$ of the cells had an expected frequency lower than 5 (Table 2).
3.2. ADHD-PI. For symptoms of ADHD-PI, the top 5 herbs with the highest composition rate were P.C (8.36\%), P.G (7.69\%), G.V (6.35\%), P.T (5.35\%), and A.G (5.02\%) (Figure 5).

Compared to the rest of the prescriptions, other than those of ADHD-PI PEHP, the usage of P.C, P.G, P.T, A.G, A.M, R.G, A.C, L.P, and Z.J for ADHD-PI was statistically significant at 0.01 and that of G.V, C.R, and B.C was significant at 0.05 . The usage of A.L and P.L was not statistically 


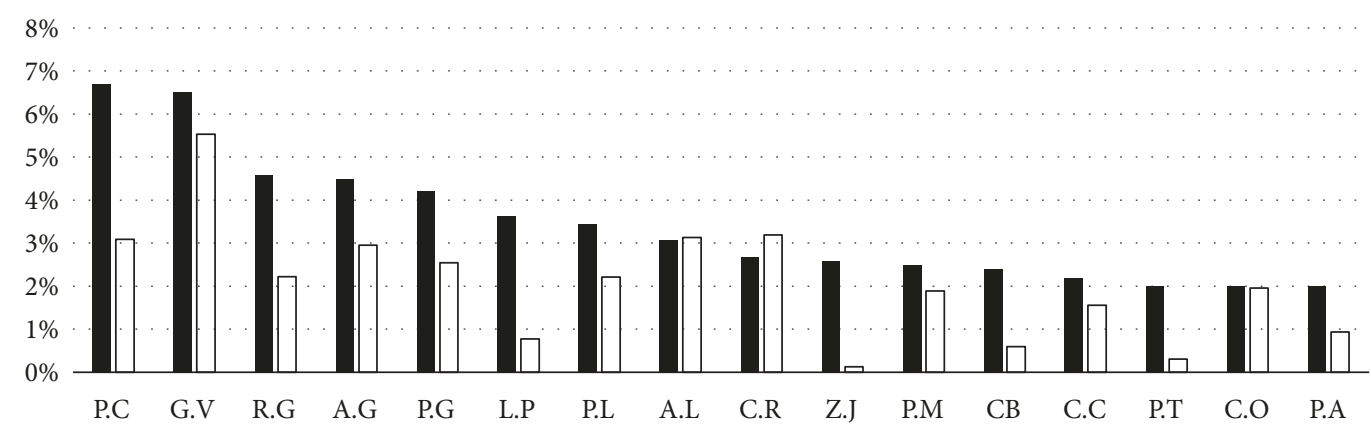

- ADHD-PHI

$\square$ ADHD-PHI et al.

P.C: Poria cocos, G.V: Glycyrrhiza uralensis, R.G: Rehmannia glutinosa, A.G: Angelica gigas, P.G: Panax

ginseng, L.P: Liriope platyphylla, P.L: Paeonia lactiflora, A.L: Atractylodes lancea, C.R: Citrus reticulata, Z.J:

Ziziphus jujuba, P.M: Pinellia ternata, C.C: Coptis chinensis, P.T: Polygala tenuifolia, C.O: Cnidium officinale,

P.A: Phellodendron amurense

FIGURE 4: Comparison of composition rate for herbs deemed potentially effective for hyperactivity attention-deficit/hyperactivity disorder type (ADHD-PHI).

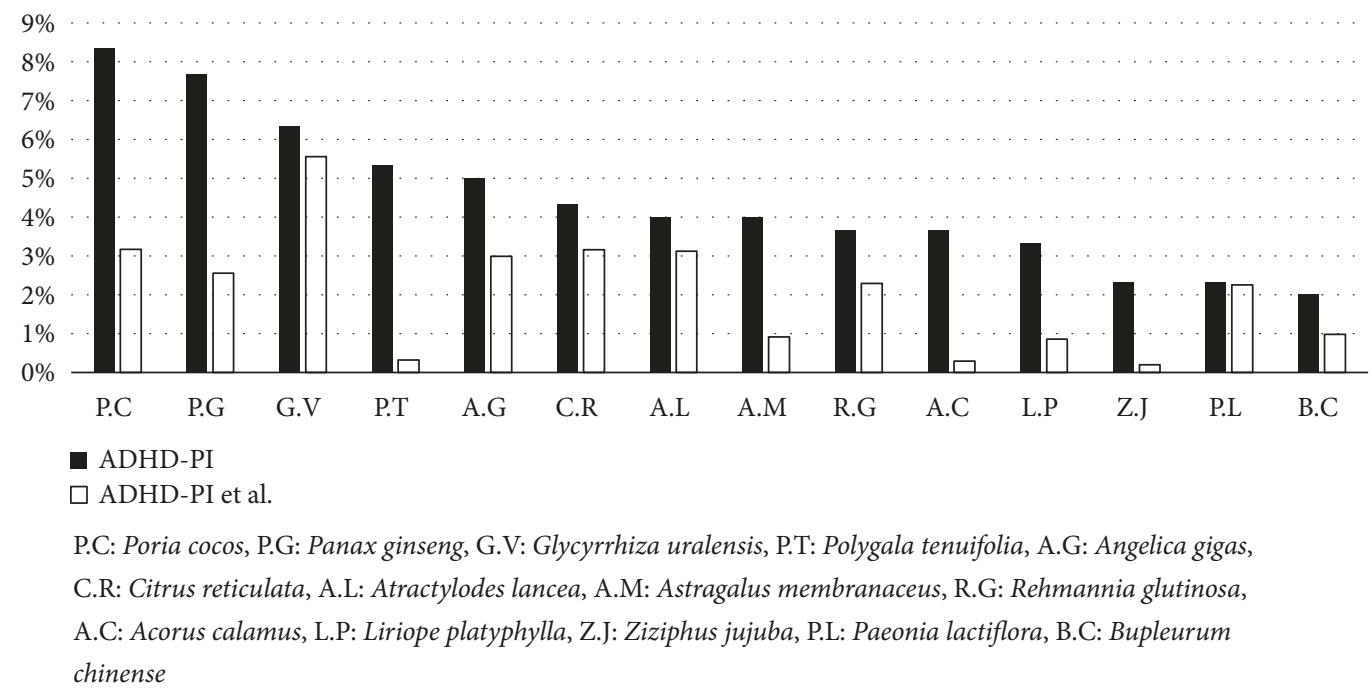

FIGURE 5: Comparison of composition rate for herbs deemed potentially effective for attention-deficit attention-deficit/hyperactivity disorder type (ADHD-PI).

significant for ADHD-PI. For P.C, P.G, A.G, C.R, A.L, R.G, and P.L, Yate's continuity correction chi-squared value was used, while the Fisher's exact test was used for P.T, A.M, A.C, L.P, Z.J, and B.C. Based on their chi-squared values, P.G (52.90) and P.C (48.26) were found to have been used specifically for ADHD-PI. The chi-squared values of P.T (266.99), A.C (137.65), and Z.J (83.39) were high, but the authors decided to exclude them from the ranking order of significance because Fisher's exact test was used (Table 3).

\section{Discussion}

By reviewing the results of the analysis, 12 out of the 156 herbs comprising the ADHD-PHI PEHP and 12 out of the 69 herbs comprising the ADHD-PI PEHP were determined to be significant for further experiments. Figures 4 and 5 showed that the statistical significance tended to present more clearly when considering the same medicinal herb, and the difference in composition rates was large between ADHD PEHP and the remaining prescriptions included in the 3833 prescriptions in Donguibogam. Moreover, when the difference in composition rates was too close to be determined, statistical tests became a useful standard of judgement. For example, G.V and P.M of ADHD-PHI and G.V and C.R of ADHD-PI were included in the list of potential herbs for ADHD treatment. On the other hand, A.L, C.R, and C.O of ADHD-PHI and A.L and P.L of ADHD-PI, which would have been selected in previous studies using simple frequency counting, were excluded.

The authors presented the order of importance of selected herbal medicines using statistics (chi-squared values). The 


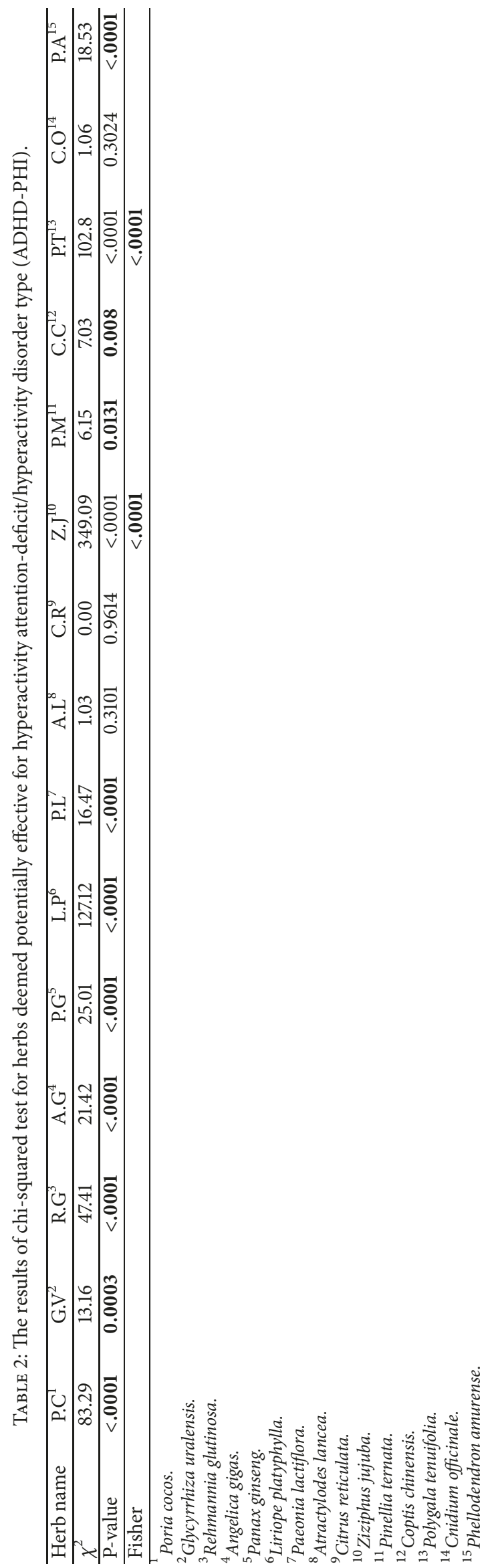




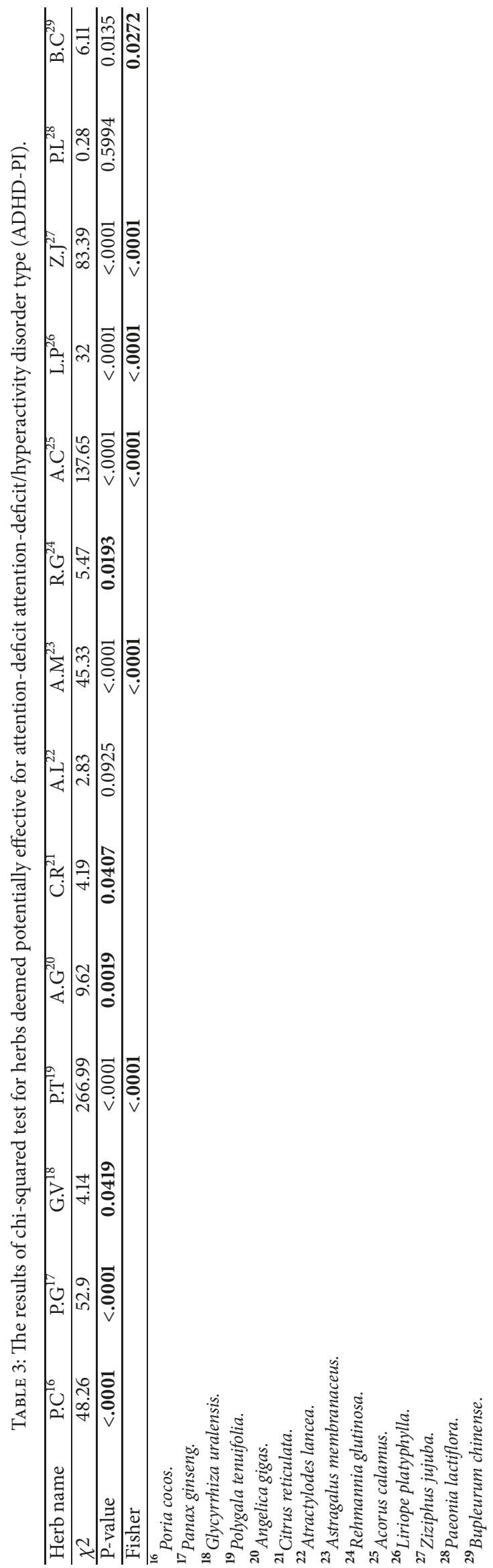


aforementioned points indicated that the methodology of this study was more systematic and efficient in selection.

To reflect the characteristic of symptomatic treatment in Korean medicine, the study was conducted separately for ADHD-PHI and ADHD-PI. The results show that 8 herbs (P.C, G.V, P.G, P.T, A.G, R.G, L.P, and Z.J) were selected with statistical significance for both subtypes of ADHD. However, 4 herbs (P.L, P.M, C.C, and P.A) were significant only for ADHD-PHI, while the other 4 (C.R, A.M, A.C, and B.C) were significant only for ADHD-PI. For each subtype of ADHD, there were 4 herbs that did not apply to the other subtype, which validated the approach in Korean medicine to consider different symptoms as separate diseases.

Since this study was an analysis of original literature, its limitation was that it needed experimental verification. However, this study remains insightful by presenting a more efficient text mining method using statistical analysis. This may be the stepping stone in the future to utilize more diverse statistical tools of analysis of original research in Korean medicine.

\section{Conclusions}

P.C, G.V, R.G, A.G, P.G, L.P, P.L, Z.J, P.M, C.C, P.T, and C.O were selected as herbs showing a high probability of effectively treating ADHD-PHI. Simultaneously, P.C, P.G, G.V, P.T, A.G, C.R, A.M, R.G, A.C, L.P, Z.J, and B.C were selected as herbs with a high probability of effectively treating ADHD-PI.

Furthermore, this study presented the chi-squared test as a new method for obtaining medicinal herbs that are useful for treating ADHD.

\section{Data Availability}

The data used to support the findings of this study are included within the article.

\section{Conflicts of Interest}

The authors declare that there are no conflicts of interest regarding the publication of this paper.

\section{Acknowledgments}

This work was supported by the Basic Science Research Program of the National Research Foundation of Korea (NRF-2018R1D1A1B07041302).

\section{References}

[1] American Psychiatric Association, Diagnostic and Statistical Manual of Mental Disorders, American Psychiatric Association, Washington, DC, USA, 5th edition, 2013.

[2] S. R. Raman, K. K. C. Man, S. Bahmanyar et al., "Trends in attention-deficit hyperactivity disorder medication use: a retrospective observational study using population-based databases," The Lancet Psychiatry, vol. 5, no. 10, pp. 824-835, 2018.
[3] V. Simon, P. Czobor, S. Bálint, Á. Mészáros, and I. Bitter, "Prevalence and correlates of adult attention-deficit hyperactivity disorder: meta-analysis," The British Journal of Psychiatry, vol. 194, no. 3, pp. 204-211, 2009.

[4] X. U. Guifeng, “Twenty-Year Trends in Diagnosed AttentionDeficit/Hyperactivity Disorder Among US Children and Adolescents, 1997-2016," 1-2, 2018.

[5] C. J. Bachmann, L. P. Wijlaars, L. J. Kalverdijk et al., "Trends in ADHD medication use in children and adolescents in five western countries, 2005-2012," European Neuropsychopharmacology, vol. 27, no. 5, pp. 484-493, 2017.

[6] Ø. Karlstad, H. Zoëga, K. Furu et al., "Use of drugs for ADHD among adults - a multinational study among 15.8 million adults in the Nordic countries," European Journal of Clinical Pharmacology, vol. 72, no. 12, pp. 1507-1514, 2016.

[7] K. C. K. Man, P. Ip, Y. F. Hisa et al., "Attention-deficit/hyperactivity disorder (ADHD) drug prescribing trend is increasing among school-aged children and adolescents," Journal of Attention Disorders, vol. 37, pp. 711-718, 2017.

[8] S. Park, "Prevalence, correlates and comorbidities of dsm-iv psychiatric disorders in children in seoul, korea," Asia-Pacific Journal of Public Health, vol. 27, Article ID 1945, 2015.

[9] M. Hong, Y. S. Kwack, Y.-S. Joung et al., "Nationwide rate of attention-deficit hyperactivity disorder diagnosis and pharmacotherapy in Korea in 2008-2011," Asia-Pacific Psychiatry, vol. 6, pp. 379-385, 2014.

[10] G. M. Kapalka, Nutritional and Herbal Therapies for Children And Adolescents: A Handbook for Mental Health Clinicians, 2010.

[11] S. Pliszka, "Practice parameter for the assessment and treatment of children and adolescents with attention-deficit/hyperactivity disorder," Journal of the American Academy of Child \& Adolescent Psychiatry, vol. 46, pp. 894-919, 2007.

[12] J. Biederman, T. Spencer, and T. Wilens, "Evidence-based pharmacotherapy for attention-deficit hyperactivity disorder," The International Journal of Neuropsychopharmacology, vol. 7, no. 1, pp. 77-96, 2004.

[13] M. Lerner and T. Wigal, "Long-term safety of stimulant medications to treat children with ADHD," Journal of Psychosocial Nursing and Mental Health Services, vol. 46, no. 8, pp. 38-48, 2008.

[14] R. A. Barkley, "Global issues related to the impact of untreated attention-deficit/ hyperactivity disorder from childhood to young adulthood," Postgraduate Medical Journal, vol. 120, no. 3 , pp. $48-59,2008$.

[15] J. K. Ghuman and H. S. Ghuman, "Pharmacologic intervention for attention-deficit hyperactivity disorder in preschoolers: Is it justified?" Pediatric Drugs, vol. 15, no. 1, pp. 1-8, 2013.

[16] H. Yuan, M. Yang, X. Han, and X. Ni, “The therapeutic effect of the chinese herbal medicine, rehmanniae radix preparata, in attention deficit hyperactivity disorder via reversal of structural abnormalities in the cortex," Evidence-Based Complementary and Alternative Medicine, pp. 1-9, 2018.

[17] J. Chen, Y. Y. Chen, and X. M. Wang, "Clinical study on children attention deficit hyperactivity disorder by Jingqian granule," Zhongguo Zhong Xi Yi Jie He Za Zhi, vol. 22, no. 4, pp. 258-260, 2002.

[18] Y. W. Wong, D.-G. Kim, and J.-Y. Lee, “Traditional oriental herbal medicine for children and adolescents with ADHD: a systematic review," Evidence-Based Complementary and Alternative Medicine, vol. 2012, Article ID 520198, 15 pages, 2012. 
[19] Y. Lan, L.-L. Zhang, and R. Luo, "Attention deficit hyperactivity disorder in children: Comparative efficacy of traditional Chinese medicine and methylphenidate," Journal of International Medical Research, vol. 37, no. 3, pp. 939-948, 2009.

[20] X. Ni, Y. Zhang-James, X. Han, S. Lei, J. Sun, and R. Zhou, "Traditional chinese medicine in the treatment of ADHD: a review," Child and Adolescent Psychiatric Clinics of North America, vol. 23, no. 4, pp. 853-881, 2014.

[21] X.-Q. Ni, X.-M. Han, D.-Q. Yin, and C.-Q. Liu, "Study on medication regularity of traditional Chinese medicines in treating attention deficit hyperactivity disorder based on data mining," Zhongguo Zhong Yao Za Zhi, vol. 40, no. 6, pp. 1185-1191, 2015.

[22] J.-H. Park, J.-H. Park, J.-H. Kim et al., "A Study on the Oriental - medical Understanding about Inattention, Hyperactivity symptom in ADHD(attention Deficit Hyperactivity Disorder)-Within Don yui bo gam Book," Journal of Oriental Neuropsychiatry, vol. 15, p. 12, 2004.

[23] J.-H. Park, J.-H. Park, J.-H. Kim et al., "A Study on the oriental medical understanding about inattention, hyperactivity symptom in adhd(attention deficit hyperactivity disorder)-within don yui bo gam book," Journal of Oriental Neuropsychiatry, vol. 15, pp. 19-24, 2004.

[24] S. P. Kellar and E. A. Kelvin, Munro's Statistical Methods for Health Care Research, vol. 28, Wolters Kluwer: Koonja, 2017. 


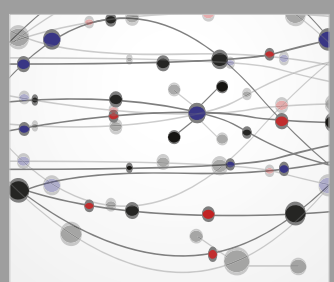

The Scientific World Journal
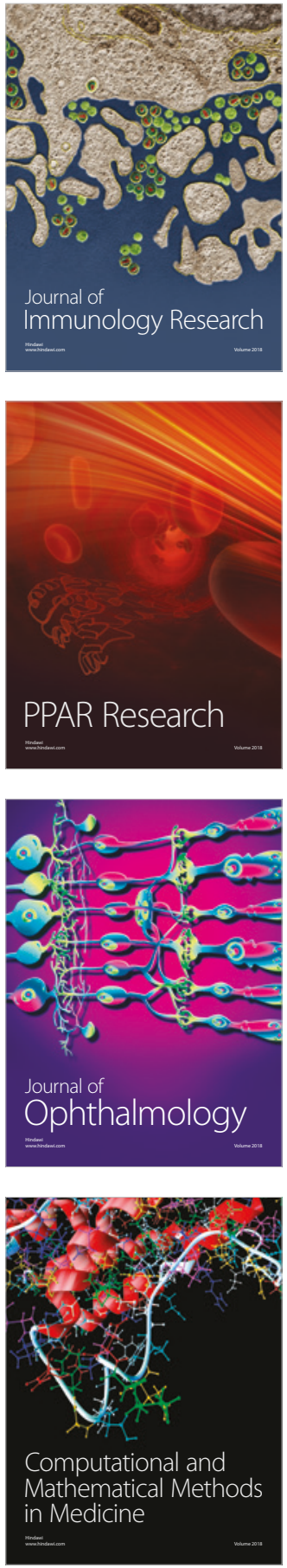

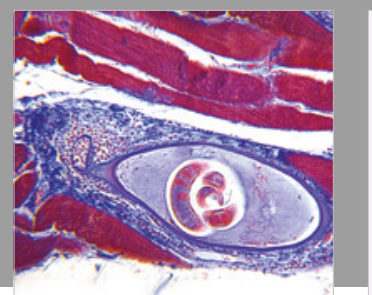

Gastroenterology Research and Practice

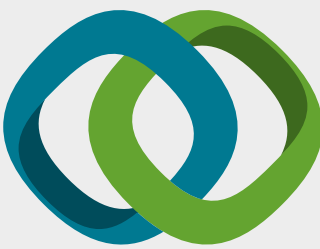

\section{Hindawi}

Submit your manuscripts at

www.hindawi.com
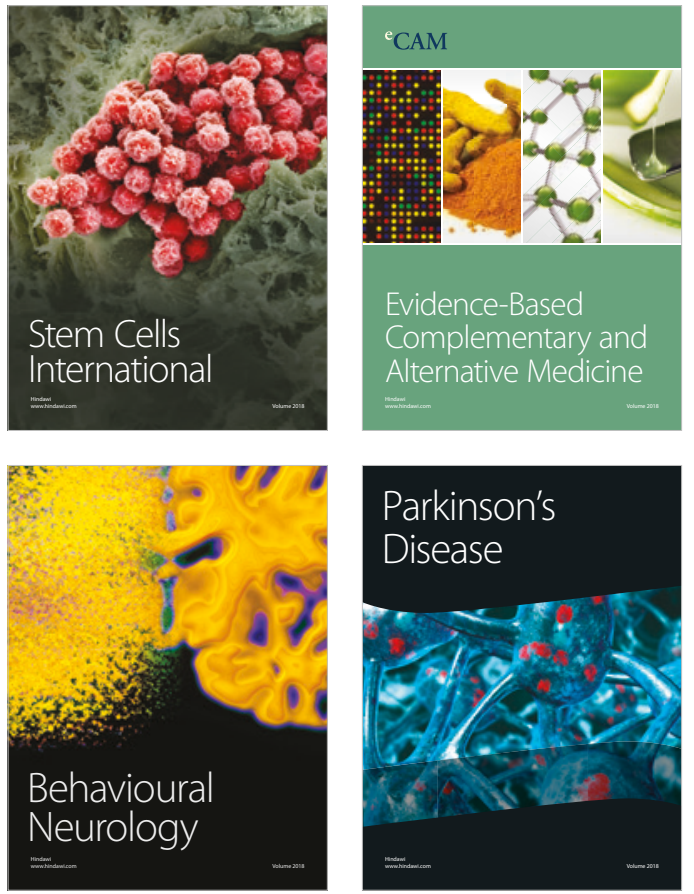

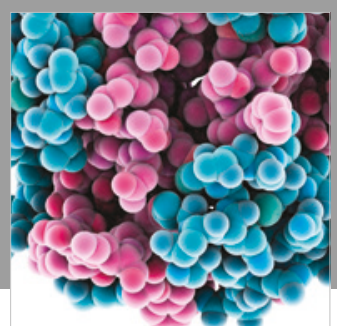

ournal of

Diabetes Research

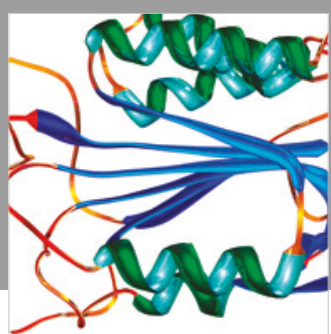

Disease Markers
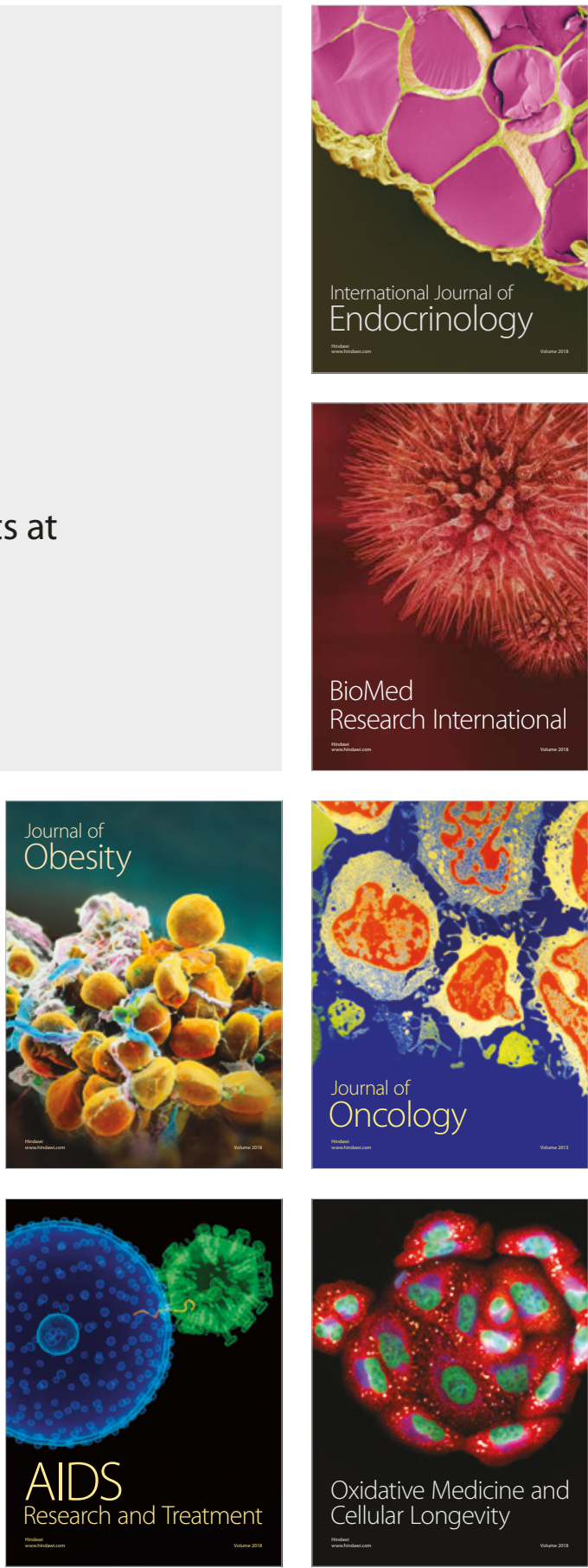